\title{
THE FARADAY 2-FORM IN EINSTEIN-WEYL GEOMETRY
}

\author{
DAVID M. J. CALDERBANK
}

\begin{abstract}
On a conformal manifold, a compatible torsion free connection $D$ need not be the Levi-Civita connection of a compatible Riemannian metric. The local obstruction is a real 2-form $F^{D}$, the Faraday curvature. It is shown that, except in four dimensions, $F^{D}$ necessarily vanishes if it is divergence free. In four dimensions another differential operator may be applied to $F^{D}$ to show that an Einstein-Weyl 4-manifold with selfdual Weyl curvature also has selfdual Faraday curvature and so is either Einstein or locally hypercomplex. More generally, the Bach tensor and the scalar curvature are shown to control the selfduality of $F^{D}$. Finally, the constancy of the sign of the scalar curvature on compact Einstein-Weyl 4-manifolds [24] is generalised to higher dimensions. The scalar curvature need not have constant sign in dimensions two and three.
\end{abstract}

\section{Introduction}

A Weyl manifold is a conformal manifold equipped with a compatible torsionfree connection. Such connections correspond bijectively to covariant derivatives on a real line bundle, which will be called Weyl derivatives. The curvature of a Weyl derivative $D$ is a real 2-form $F^{D}$, whose vanishing implies that the induced torsion-free connection is locally the Levi-Civita connection of a compatible Riemannian metric. This is the original gauge theory introduced by Hermann Weyl [28] as a geometrisation of classical electromagnetism: $F^{D}$ is the Faraday 2-form representing the electromagnetic field.

There has been a resurgence of interest in Weyl geometry recently, not only because of the original motivation, but also because of a number of more surprising links with geometry and mathematical physics. The germinal idea to unify general relativity and electromagnetism led naturally to the notion of an Einstein-Weyl manifold, in which the symmetric trace free part of the Ricci tensor of the Weyl connection is assumed to vanish. However, the geometry of Einstein-Weyl manifolds initially attracted particular interest in three dimensions, rather than four, thanks to work of Cartan [7], and Hitchin [18], who showed that the Einstein-Weyl condition is the integrability condition for a complex structure on the space of oriented geodesics of a three dimensional Weyl manifold. This provides a twistor correspondence between

Received October 26, 1998. 
Einstein-Weyl 3-manifolds and complex surfaces containing rational curves with normal bundle $\mathscr{O}(2)$. Subsequently, Einstein-Weyl manifolds have been much studied in all dimensions [5], [6], [9], [14], [22], [23], [24], [25], [26], but the three dimensional case retains a special aura, thanks to the above twistor construction, and the link with selfdual conformal 4-manifolds [19].

In four dimensions, locally hypercomplex 4-manifolds are Einstein-Weyl [23], and this class of Einstein-Weyl geometries has recently been linked to local heterotic geometry [27]. One of the main aims of this paper is to prove that every Einstein-Weyl 4-manifold with selfdual Weyl tensor is either Einstein or locally hypercomplex. This local result, like several of the results in this paper, was previously only known in the compact case.

After setting up a framework for studying Weyl geometry in a gauge invariant way, I present a very simple result which shows that, except in four dimensions, the vanishing of the divergence of $F^{D}$ (with respect to the Weyl connection) is enough to ensure that $F^{D}$ itself vanishes. A consequence of this is that $F^{D}$ necessarily vanishes on scalar flat Einstein-Weyl $n$-manifolds, for $n \neq 4$. In four dimensions, scalar-flatness only implies that $F^{D}$ is source-free, i.e., coclosed; in other words its selfdual and antiselfdual parts $F_{+}^{D}$ and $F_{-}^{D}$ are both closed. I next show, in section 4 , that the vanishing of one of $F_{+}^{D}$ and $F_{-}^{D}$ implies that the Bach tensor of the underlying conformal structure is identically zero. The converse also holds, as long as we continue to assume vanishing scalar curvature. These results are established by giving a formula for the Bach tensor on an Einstein-Weyl 4-manifold (Theorem 4.4) generalising a formula of [24] to the local setting.

Also in section 4, I present some identities relating to the Bach tensor, which may be used to verify (locally) that it vanishes when the Weyl tensor is (anti)selfdual. This fact is not used in the proof of Theorem 4.4, nor is it new: see [3], [8] for the statement, and [12] for a proof using the normal conformal connection. However, these calculations do provide motivation for considering the Bach tensor in a local context, and they are not readily accessible in the literature, so I hope it will be of some interest to sketch them briefly here.

In [9], Eastwood and Tod have shown that the Faraday 2-form vanishes on conformally flat Einstein-Weyl manifolds. In section 5, I reformulate their result and demonstrate that the same ideas may be used to prove that half conformally flat Einstein-Weyl 4-manifolds have (anti)selfdual Faraday curvature, and are therefore Einstein or locally hypercomplex by [23]. This was previously only known in the compact case [14], [24] and shows that the twistor theory of Pedersen and Swann [23] deals with all Einstein-Weyl structures on an (anti)selfdual conformal 4-manifold.

I end the paper with a discussion of the sign of the scalar curvature on compact Einstein-Weyl manifolds. This was shown to be constant in four di- 
mensions by Pedersen and Swann [24]. I show that the same is true in higher dimensions, but not in dimensions two and three, contrary to previous assertions [24], [25]. The higher dimensional result follows by applying the maximum principle to a formula in [24], while the low dimensional counterexamples can be found in [6], [25], [26].

ACKNOWLEDGMENTs. Several of the results in this paper arose during the preparation of a survey paper on Einstein-Weyl geometry in collaboration with Henrik Pedersen [5], and I gratefully acknowledge many useful and instructive conversations with him. The approach taken in section 5 is strongly influenced by numerous discussions with Tammo Diemer on linear field theories in conformal geometry, and I am likewise indebted to him. Finally, I would like to thank Paul Gauduchon, Henrik Pedersen and Andrew Swann for discussing with me the sign of the scalar curvature.

\section{Weyl derivatives}

In order to carry out computations in a gauge invariant way, it is convenient to work with densities. If $V$ is a real $n$-dimensional vector space and $w$ any real number, then the oriented one dimensional linear space $L^{w}=L^{w}(V)$ carrying the representation $A \mapsto|\operatorname{det} A|^{w / n}$ of $\mathrm{GL}(V)$ will be called the space of densities of weight $w$ or $w$-densities. It can be constructed canonically as the space of maps $\rho:\left(\Lambda^{n} V\right) \backslash 0 \rightarrow$ R such that $\rho(\lambda \omega)=|\lambda|^{-w / n} \rho(\omega)$ for all $\lambda \in \mathrm{R}^{\times}$and $\omega \in\left(\Lambda^{n} V\right) \backslash 0$.

Definition 2.1. Suppose $M$ is any $n$-manifold. Then the density line bundle $L^{w}=L^{w}(T M)$ of $M$ is defined to be the bundle whose fibre at $x \in M$ is $L^{w}\left(T_{x} M\right)$. Equivalently it is the associated bundle $\mathrm{GL}(M) \times_{\mathrm{GL}(n)} L^{w}(n)$ where $\operatorname{GL}(M)$ is the frame bundle of $M$ and $L^{w}(n)$ is the space of $w$-densities of $\mathrm{R}^{n}$.

The density bundles are oriented (hence trivialisable) real line bundles, but there is no preferred trivialisation. Sections of $L=L^{1}$ may be thought of as scalar fields with dimensions of length. This geometric dimensional analysis may also be applied to tensors: the tensor bundle $L^{w} \otimes(T M)^{j} \otimes\left(T^{*} M\right)^{k}$ (and any subbundle, quotient bundle, element or section) will be said to have weight $w+j-k$, or dimensions of [length ${ }^{w+j-k}$.

Note that sections of $L^{-n}$ may be invariantly integrated, and that an orientation of $M$ defines an isomorphism between $L^{-n}$ and $\Lambda^{n} T^{*} M$. Also note that $L^{w_{1}} \otimes L^{w_{2}}$ is canonically isomorphic to $L^{w_{1}+w_{2}}$ and $L^{0}$ is the trivial bundle. When tensoring a vector bundle with some $L^{w}$, I shall often omit the tensor product sign. A nonvanishing (usually positive) section of $L^{1}$ (or $L^{w}$ for $w \neq 0$ ) will be called a length scale or gauge (of weight $w$ ). 
Definition 2.2. A Weyl derivative is a covariant derivative $D$ on $L^{1}$. It induces covariant derivatives on $L^{w}$ for all $w$. The curvature of $D$ is a real 2-form $F^{D}$ which will be called the Faraday curvature or Faraday 2-form.

If $F^{D}=0$ then $D$ is said to be closed. It follows that there are local length scales $\mu$ with $D \mu=0$. If such a length scale $\mu$ exists globally then $D$ is said to be exact. Conversely, a length scale $\mu$ induces an exact Weyl derivative $D^{\mu}$ such that $D^{\mu} \mu=0$. Note that Weyl derivatives form an affine space modelled on the linear space of 1-forms, while closed and exact Weyl derivatives are affine subspaces modelled on the linear spaces of closed and exact 1-forms respectively.

A gauge transformation on $M$ is a positive function $e^{f}$ which rescales a gauge $\mu \in \mathrm{C}^{\infty}\left(M, L^{w}\right)$ to give $e^{w f} \mu$. If $D$ is any Weyl derivative, then $D=$ $D^{\mu}+\omega^{\mu}$ for the 1 -form $\omega^{\mu}=\mu^{-1} D \mu$, and consequently, $\omega^{e^{f} \mu}=\omega^{\mu}+d f$.

The notion of density allows one to define a conformal structure, not as an equivalence class of inner products, but a genuine inner product.

DEFINITION 2.3. A conformal structure on a manifold $M$ is a normalised $L^{2}$ valued inner product on $T M$. More precisely it is a section $\subset \in$ $\mathrm{C}^{\infty}\left(M, L^{2} S^{2} T^{*} M\right)$ which is everywhere positive definite. The conformal inner product of tangent vector fields $X, Y$ will be denoted $\langle X, Y\rangle$, and is a section of $L^{2}$.

A conformal structure may also be viewed as a metric on the weightless tangent bundle $L^{-1} T M$. The normalisation condition states that $|\operatorname{det} \mathrm{c}|=1$, which makes sense since the densities of $L^{-1} T M$ are canonically trivial.

Conformal structures and Weyl derivatives fit together very well: for instance, Riemannian geometry is the geometry of an exact Weyl derivative on a conformal manifold. The existence and uniqueness of the Levi-Civita connection is a special case of the following central result.

Theorem 2.4 (The Fundamental Theorem of Conformal Geometry). [28] On a conformal manifold $M$ there is an affine bijection between covariant derivatives on $L^{1}$ and torsion free connections on $T M$ preserving the conformal structure. More explicitly, the covariant derivative on $T M$ is determined from the one on $L^{1}$ by the Koszul formula

$$
\begin{aligned}
2\left\langle D_{X} Y, Z\right\rangle= & D_{X}\langle Y, Z\rangle+D_{Y}\langle X, Z\rangle-D_{Z}\langle X, Y\rangle \\
& +\langle[X, Y], Z\rangle-\langle[X, Z], Y\rangle-\langle[Y, Z], X\rangle,
\end{aligned}
$$

where $X, Y, Z$ are vector fields and the conformal inner products are sections of $L^{2}$. 
A conformal manifold equipped with a Weyl derivative $D$ is called a Weyl manifold. Henceforth $D$ will be identified with the induced connection on $T M$ and all associated bundles.

It is sometimes useful to compare two Weyl derivatives on a conformal manifold: suppose that $\tilde{D}=D+\gamma$, where $\gamma$ is a 1-form, and suppose that the induced connections on $L^{w-1} T M$ are related by $\tilde{D}=D+\Gamma$ for some $\operatorname{co}(T M)$-valued 1-form $\Gamma$. Then $\Gamma$ is given in terms of $\gamma$ by the linearised Koszul formula:

$$
\Gamma_{X}=w \gamma(X) \text { id }+\gamma \triangle X, \quad \text { where } \quad(\gamma \triangle X)(Y)=\gamma(Y) X-\langle X, Y\rangle \gamma .
$$

Here free use is being made of the natural isomorphism $\sharp: T^{*} M \rightarrow L^{-2} T M$ given by the conformal structure.

A basic formula in Weyl geometry (and indeed Riemannian geometry) is the following decomposition of the curvature $R^{D, w}$ of $D$ on $L^{w-1} T M$ :

$$
R_{X, Y}^{D, w}=W_{X, Y}+w F^{D}(X, Y) \mathrm{id}-r^{D}(X) \triangle Y+r^{D}(Y) \triangle X .
$$

Here $W_{X, Y}$ is the Weyl curvature (independent of $D$ ), $F^{D}$ is the Faraday curvature (independent of $\mathrm{C}$ ), and $r^{D}$ is a section of $L^{-2}$ End $T M$ called the normalised Ricci tensor. (Convention: $R_{X, Y}^{D, w}=D_{X, Y}^{2}-D_{Y, X}^{2}=\left[D_{X}, D_{Y}\right]-$ $\left.D_{[X, Y]}.\right)$

The normalised Ricci tensor may be computed from $R^{D, w}$ by taking a trace (since $W$ is tracefree) — such a trace does not necessarily produce a symmetric tensor. An alternative description of $r^{D}$ is given by its decomposition under the orthogonal group:

$$
r^{D}=r_{0}^{D}+\frac{1}{2 n(n-1)} \operatorname{scal}^{D} \mathrm{id}-\frac{1}{2} F^{D},
$$

where $r_{0}^{D}$ is symmetric and tracefree and scal ${ }^{D}$ is the scalar curvature of $D$ (which is a section of $L^{-2}$ ).

Two of the results in this paper are based on the idea of twisting a complex of differential operators by a connection. The most familiar example of this is the deRham complex. If we have an auxiliary bundle $E$, then a connection on $E$ induces a sequence of twisted exterior derivatives on $E$-valued differential forms. This sequence is no longer a complex in general: the composite of two twisted exterior derivatives is given by the natural action of the curvature of the connection.

This idea will be applied to the density bundles and Weyl derivatives, but using other differential complexes. The first such result is a very simple one. 
Instead of considering the deRham complex of exterior derivatives

$$
M \times \mathrm{R} \stackrel{d}{\rightarrow} T^{*} M \stackrel{d}{\rightarrow} \Lambda^{2} T^{*} M \rightarrow \cdots
$$

consider the adjoint complex of exterior divergences

$$
L^{-n} \stackrel{\delta}{\leftarrow} L^{-n} T M \stackrel{\delta}{\leftarrow} L^{-n} \Lambda^{2} T M \leftarrow \cdots
$$

Here $\delta=\operatorname{tr} D$, where $D$ is any torsion free connection and the trace is with respect to the first entry: thus $\delta$ is minus the formal adjoint of $d$. Both are complexes of invariant operators. Using the conformal structure, we obtain a complex of conformally invariant operators:

$$
L^{-n} \stackrel{\delta}{\leftarrow} L^{2-n} T^{*} M \stackrel{\delta}{\leftarrow} L^{4-n} \Lambda^{2} T^{*} M \leftarrow \cdots
$$

It is this complex which I shall twist by a Weyl derivative.

Proposition 2.5. Let $D$ be a Weyl derivative on a conformal manifold $M$. Then $\left(\delta^{D}\right)^{2} F^{D}=-(n-4)\left|F^{D}\right|^{2}$. If $n \neq 4$ it follows that $\delta^{D} F^{D}=0$ iff $F^{D}=0$.

Proof. Since $F^{D}$ is a 2-form (of weight -2 ), the exterior divergence has been twisted by $D$ on $L^{n-4}$. Suppose that $D=D^{0}+\omega$ on $L^{1}$. Then for any 2 -form $\phi$,

$$
\delta^{D^{0}+\omega} \phi=\delta^{D^{0}} \phi+(n-4) \phi(\omega, .) .
$$

If $D^{0}$ is closed, it follows that

$$
\begin{aligned}
\left(\delta^{D}\right)^{2} \phi & =\delta^{D^{0}+\omega} \delta^{D^{0}+\omega} \phi \\
& =\delta^{D^{0}} \delta^{D^{0}} \phi+(n-4)\left(\delta^{D^{0}}(\phi(\omega, .))+\left(\delta^{D^{0}} \phi\right)(\omega)\right) \\
& =(n-4) \operatorname{tr} \phi\left(D^{0} \omega, .\right),
\end{aligned}
$$

where $\left(\delta^{D^{0}}\right)^{2}=0$ because $L^{n-4}$ may be locally trivialised by a $D^{0}$-parallel section (equivalently, when we twist a complex by a flat connection, it remains a complex). Therefore

$$
\left(\delta^{D}\right)^{2} \phi=(n-4) \sum_{i, j} D_{e_{i}}^{0} \omega\left(e_{j}\right) \phi\left(e_{j}, e_{i}\right)=(n-4) \sum_{i<j} d \omega\left(e_{i}, e_{j}\right) \phi\left(e_{j}, e_{i}\right)
$$

and $d \omega=F^{D}$ since $D^{0}$ is closed. Substituting $\phi=F^{D}$ proves the proposition.

As in the Riemannian case, the second Bianchi identity is a useful tool in Weyl geometry. The differential Bianchi identity for the Weyl derivative $D$ 
on $L^{1}$ gives the simple fact that $d F^{D}=0$, while for the induced covariant derivative on $L^{w-1} T M$, we have $d^{D} R^{D, w}=0$, where $R^{D, w}$ is viewed as an endomorphism valued 2-form. In particular:

$$
\left(D_{X} R^{D, 0}\right)_{Y, Z} U+\left(D_{Z} R^{D, 0}\right)_{X, Y} U+\left(D_{Y} R^{D, 0}\right)_{Z, X} U=0 .
$$

If we take the trace of this identity as a function of $Z$, and separate out the trace using the conformal structure and (1), then we obtain, for $n>2$, the contracted Bianchi identities:

$$
\begin{aligned}
\operatorname{tr} D W_{X, Y} U & =(n-3) C_{X, Y}^{D} U \\
C_{X, e_{i}}^{D} e_{i} & =0 .
\end{aligned}
$$

Here $C_{X, Y}^{D}=\left(d^{D} r^{D}\right)_{X, Y}=\left(D_{X} r^{D}\right)(Y)-\left(D_{Y} r^{D}\right)(X)$ is the Cotton-York tensor of $D$, and there is an implied summation over the weightless orthonormal basis $e_{i}$.

\section{Einstein-Weyl geometry}

A Weyl manifold of dimension $n>2$ is said to be Einstein-Weyl iff $r_{0}^{D}=0$. This class of Weyl manifolds has been extensively studied (see [5] and references therein). The Einstein-Weyl manifolds with exact Weyl derivative are precisely the (homothety classes of) Einstein metrics, and the closed EinsteinWeyl manifolds are "locally conformally Einstein". There are also many examples with nonzero Faraday curvature [4], [19], [21], [23], [24], [25].

The contracted Bianchi identity gives an important relationship between the Faraday and scalar curvatures. Writing out (4) explicitly gives,

$$
\operatorname{div}^{D} r_{0}^{D}-\frac{1}{2 n} D \operatorname{scal}^{D}+\frac{1}{2} \delta^{D} F^{D}=0,
$$

from which we immediately have the following.

Proposition 3.1. [25], [14] Suppose $M$ is Einstein-Weyl of dimension $n>2$. Then $D \mathrm{scal}^{D}-n \delta^{D} F^{D}=0$.

When $n=2$, we define Einstein-Weyl manifolds by this identity [6], generalising the metrics of constant scalar curvature.

A consequence of the Einstein-Weyl Bianchi identity is the following formula, given by Pedersen and Swann in the compact case:

Proposition 3.2. (cf. [24]) Let $M$ be an n-dimensional Einstein-Weyl manifold. Then $\Delta^{D} \mathrm{scal}^{D}=-n(n-4)\left|F^{D}\right|^{2}$.

Proof. Here $\Delta^{D}=\operatorname{tr} D^{2}$, so this follows by applying $D$ to the formula in 3.1 and taking trace, where Proposition 2.5 is used to compute $\operatorname{tr} D\left(\delta^{D} F^{D}\right)=$ $\left(\delta^{D}\right)^{2} F^{D}$. 
I will make use of this later.

THEOREM 3.3. If $\left(M^{n}, D\right)$ is Einstein-Weyl, the following are equivalent:

(i) Either $D$ is closed or $n=4, M$ is noncompact and $F^{D}$ is harmonic.

(ii) $\delta^{D} F^{D}=0$.

(iii) $D \mathrm{scal}^{D}=0$.

(iv) Either $D$ is exact or $\mathrm{scal}^{D}$ is identically zero.

Before proving this theorem, let me make some remarks. There are two important consequences to be extracted from these equivalences. The first of these is the fact that if $D$ is closed then $D$ is exact or $\operatorname{scal}^{D}=0$. This is not new: it was established by Gauduchon [14] using 3.1, and by Higa [16], using the contracted Bianchi identity on the universal cover (which is Einstein). The second consequence is that if $\mathrm{scal}^{D}=0$ then $D$ is closed, except in the local four dimensional case. This was previously only known for compact manifolds [14], [25]. The new part of the above theorem follows easily from 2.5 .

Proof. (ii) and (iii) are equivalent by 3.1 , and clearly (iii) $\Longrightarrow$ (iv) $\Longrightarrow$ (ii) or (iii). Also (i) immediately implies (ii)—note that the divergence on 2-forms is conformally invariant in four dimensions-so it remains to prove the converse. This follows from 2.5 unless $M$ is four dimensional, in which case $F^{D}$ is an exact coclosed 2-form, and so it is certainly harmonic. If $M$ is compact, an exact coclosed 2-form necessarily vanishes: write $F^{D}=d \gamma$ and integrate the section $\left|F^{D}\right|^{2}$ of $L^{-4}$ by parts.

\section{Four dimensions and the Bach tensor}

The four dimensional case is singled out by Theorem 3.3 for good reason: it is only in four dimensions that the divergence is conformally invariant on 2-forms. Closely related to this is the fact that the bundle of 2-forms is not irreducible, but splits into the selfdual and antiselfdual 2-forms. Correspondingly, the Faraday 2-form decomposes as $F^{D}=F_{+}^{D}+F_{-}^{D}$.

As shown by Pedersen and Swann [23], hypercomplex manifolds provide examples of scalar flat four dimensional Einstein-Weyl manifolds with nonzero Faraday curvature. In this case, if we fix the orientation by taking the complex structures to be antiselfdual, then the Faraday 2-form and Weyl tensor are both selfdual. [There are explicit examples amongst the Pedersen/LeBrun metrics: these are U(2)-symmetric selfdual conformal structures, which are both conformally Einstein (with nonzero scalar curvature) [22] and conformally scalar flat Kähler (with antiselfdual complex structure) [20]. They may be constructed from harmonic functions ("monopoles") on $S^{3}$ or $\mathscr{H}^{3}$. Those coming from $S^{3}$ also admit a hypercomplex structure with nonzero Faraday curvature [21].] 
On the other hand, the theory in the previous section only shows that $F_{+}^{D}$ and $F_{-}^{D}$ are both closed, or equivalently, $F^{D}$ is a Maxwell field (an abelian YangMills field). It is therefore natural to ask whether the Einstein-Weyl condition implies that $F^{D}$ is an (anti)selfdual Maxwell field. We might then be able to sharpen Theorem 3.3.

In this section I will show that this question is closely related to the Bach tensor, the weight -4 symmetric traceless bilinear form which arises as the gradient of the functional

$$
\mathrm{C} \mapsto \int_{M}|W|^{2} .
$$

Here $W$ is the Weyl curvature of c, so that $|W|^{2}$ is a section of $L^{-4}$. It is well known [3] that the signature formula may be used to show that compact conformal 4-manifolds with (anti)selfdual Weyl are absolute minima of this functional. It is also known, but perhaps less widely appreciated, that there is also a local result: 4-manifolds with (anti)selfdual Weyl tensor have vanishing Bach tensor. Since I have not found a convenient reference for the local theory, I will sketch it here.

Definition 4.1. Let $M$, c, $D$ be a Weyl $n$-manifold $(n>2)$. Then the Bach tensor of $M$ is defined by the formula

$$
B^{D}(X, Y)=D_{e_{i}} C_{e_{i}, X}^{D} Y+r^{D}\left(e_{i}, W_{e_{i}, X} Y\right)
$$

where $r^{D}(X, Y)=\left\langle r^{D}(X), Y\right\rangle$ and there is an implied summation over the weightless orthonormal basis $e_{i}$.

It is not immediately clear that this is symmetric, although it is clearly tracefree by (4). The symmetry turns out to be a consequence of a contracted "third Bianchi identity". The first Bianchi identity shows that the Weyl curvature has the symmetries of a "Weyl tensor", while the second Bianchi identity shows that it lies in the kernel of a conformally invariant first order differential operator. In four dimensions, however, this first order condition is vacuous, and the Weyl curvature lies in the kernel of a conformally invariant second order differential operator. This second order operator arises from the third Bianchi identity.

All three Bianchi identities are consequences of the differential Bianchi identity for the conformal connection [12]. However, I shall prove the following directly.

Proposition 4.2. Let $D$ be any Weyl derivative a conformal manifold. Then

$$
d^{D} C_{X, Y, Z}^{D} U+r^{D}\left(X, W_{Y, Z} U\right)+r^{D}\left(Z, W_{X, Y} U\right)+r^{D}\left(Y, W_{Z, X} U\right)=0 .
$$


Here $d^{D} C^{D}$ denotes the exterior derivative of $C^{D}$ as a covector valued 2-form.

Proof. Since $C^{D}=d^{D} r^{D}$, we readily see that:

$$
\begin{aligned}
d^{D} C_{X, Y, Z}^{D}=\left(D_{X, Y}^{2} r^{D}\right)(Z)+\left(D_{Z, X}^{2} r^{D}\right)(Y)+\left(D_{Y, Z}^{2} r^{D}\right)(X) \\
\\
-\left(D_{Y, X}^{2} r^{D}\right)(Z)-\left(D_{X, Z}^{2} r^{D}\right)(Y)-\left(D_{Z, Y}^{2} r^{D}\right)(X) .
\end{aligned}
$$

Note that

$$
\left(D_{X, Y}^{2} r^{D}\right)(Z, U)-\left(D_{Y, X}^{2} r^{D}\right)(Z, U)=-r^{D}\left(R_{X, Y}^{D} Z, U\right)-r^{D}\left(Z, R_{X, Y}^{D} U\right),
$$

where $R^{D}=R^{D, 1}$, and the first term cancels with its cyclic permutations by the first Bianchi identity. For the second term we compute:

$$
\begin{aligned}
r^{D}\left(Z, R_{X, Y}^{D} U\right)= & r^{D}\left(Z, W_{X, Y} U\right)+r^{D}\left(Z,\left(r^{D}(Y) \triangle X\right) U\right) \\
& -r^{D}\left(Z,\left(r^{D}(X) \triangle Y\right) U\right)+F^{D}(X, Y) r^{D}(Z, U) \\
=\left\langle r^{D}(Z), W_{X, Y} U\right\rangle+\left\langle r^{D}(X), r^{D}(Z)\right\rangle\langle Y, U\rangle & -\left\langle r^{D}(Y), r^{D}(Z)\right\rangle\langle X, U\rangle+r^{D}(Z, Y) r^{D}(X, U) \\
& -r^{D}(Z, X) r^{D}(Y, U)+F^{D}(X, Y) r^{D}(Z, U) .
\end{aligned}
$$

On taking cyclic permutations over $X, Y, Z$, the second and third terms cancel by symmetry, and the last three terms cancel because the skew symmetric part of $r^{D}$ is $-\frac{1}{2} F^{D}$. The first term and its cyclic permutations give the stated formula.

The symmetry of the Bach tensor follows from the following properties of the Cotton-York tensor.

Proposition 4.3. Let $C^{D}$ be the Cotton-York tensor of a Weyl n-manifold. Then

$$
\begin{aligned}
C_{X, Y}^{D} Z+C_{Y, Z}^{D} X+C_{Z, X}^{D} Y & =0 \\
D_{e_{i}} C_{X, Y}^{D} e_{i}+r^{D}\left(e_{i}, W_{X, Y} e_{i}\right) & =0 .
\end{aligned}
$$

In the second formula, only the skew symmetric part of $r^{D}$ contributes.

Proof. The first part follows easily from the fact that the skew symmetric part of $r^{D}$, namely $-\frac{1}{2} F^{D}$, is closed. The second formula generalises the fact that the Cotton-York tensor on a 3-manifold is divergence free and follows immediately by taking the trace over $Z$ and $U$ of the third Bianchi identity (5). 
Putting (6) and (7) together gives

$$
\begin{aligned}
B^{D}(X, Y)-B^{D}(Y, X)= & D_{e_{i}} C_{e_{i}, X}^{D} Y-D_{e_{i}} C_{e_{i}, Y}^{D} X \\
& +r^{D}\left(e_{i}, W_{e_{i}, X} Y\right)-r^{D}\left(e_{i}, W_{e_{i}, Y} X\right) \\
= & -D_{e_{i}} C_{X, Y}^{D} e_{i}-r^{D}\left(e_{i}, W_{X, Y} e_{i}\right)=0 .
\end{aligned}
$$

From this it follows that an alternative (perhaps better) formula for the Bach tensor is:

$$
B^{D}(X, Y)=\frac{1}{2}\left(D_{e_{i}} C_{e_{i}, X}^{D} Y+D_{e_{i}} C_{e_{i}, Y}^{D} X\right)+r_{0}^{D}\left(e_{i}, W_{e_{i}, X} Y\right) .
$$

In dimension four, $B=B^{D}$ is independent of the choice of $D$. To check this, consider another Weyl derivative $\tilde{D}=D+\gamma$. Then direct calculations give the following formulae:

$$
\begin{aligned}
r^{\tilde{D}} & =r^{D}-\left(D \gamma-\gamma \otimes \gamma+\frac{1}{2}\langle\gamma, \gamma\rangle \mathrm{id}\right) \\
C_{X, Y}^{\tilde{D}} U & =C_{X, Y}^{D} U+\gamma\left(W_{X, Y} U\right) \\
B^{\tilde{D}}(X, Y) & =B^{D}(X, Y)+(n-4)\left(C_{\gamma, X}^{D} Y+C_{\gamma, Y}^{D} X+\gamma\left(W_{\gamma, X} Y\right)\right) .
\end{aligned}
$$

For $n>3$, the third Bianchi identity and the definition of the Bach tensor can be rewritten using the second Bianchi identity (3):

$$
\begin{aligned}
& \left(d^{D} \operatorname{div}^{D} W\right)_{X, Y, Z} U+r^{D}\left(Z, W_{X, Y} U\right)+r^{D}\left(Y, W_{Z, X} U\right)+r^{D}\left(X, W_{Y, Z} U\right)=0 \\
& \left(\delta^{D} \operatorname{div}^{D} W\right)_{X} Y+(n-3) r^{D}\left(e_{i}, W_{e_{i}, X} Y\right)=(n-3) B^{D}(X, Y) .
\end{aligned}
$$

$\left(d^{D}\right.$ and $\delta^{D}$ are the exterior derivative and divergence on covector valued 2forms.)

In order to interpret this, we need to choose an orientation and introduce the conformal Hodge star operator $*: L^{w+k} \Lambda^{k} T^{*} M \rightarrow L^{w+n-k} \Lambda^{n-k} T^{*} M$. In four dimensions, if we view $W$ as an endomorphism valued 2-form, and its divergence $C^{D}$ as a covector valued 2-form, then the star operator decomposes $W$ and $C^{D}$ into selfdual and antiselfdual parts. We then see that $* d^{D} \operatorname{div}^{D} W=$ $\delta^{D} \operatorname{div}^{D} * W$, and consequently, the Bach tensor may be computed from $W^{+}$ or $W^{-}$:

$$
\begin{aligned}
B(X, Y) & =2\left(\delta^{D} \operatorname{div}^{D} W^{+}\right)_{X} Y+2 r^{D}\left(e_{i}, W_{e_{i}, X}^{+} Y\right) \\
& =2\left(\delta^{D} \operatorname{div}^{D} W^{-}\right)_{X} Y+2 r^{D}\left(e_{i}, W_{e_{i}, X}^{-} Y\right) .
\end{aligned}
$$

Hence we finally have that the Bach tensor vanishes on (anti)selfdual conformal 4-manifolds.

These formulae also relate to conformally invariant second order differential operators acting on weight -2 Weyl tensors in dimension four (cf. [2], [8]). 
I will now turn to the impact of the Bach tensor on Einstein-Weyl geometry. In [24], Pedersen and Swann gave a formula for the Bach tensor of a compact Einstein-Weyl manifold in terms of the 1-form of the Weyl structure in the Gauduchon gauge. They deduced that on a compact Bach flat $(B=0)$ EinsteinWeyl 4-manifold, the Faraday 2-form necessarily vanishes.

The Bach tensor and Faraday 2-form are intimately linked even locally.

Theorem 4.4. Let $M$ be an Einstein-Weyl 4-manifold. Then

$$
\begin{aligned}
B(X, Y)=\frac{1}{24}\left(D_{X, Y}^{2} \operatorname{scal}^{D}\right. & \left.+D_{Y, X}^{2} \operatorname{scal}^{D}\right) \\
& -\left\langle F_{+}^{D}(X), F_{-}^{D}(Y)\right\rangle-\left\langle F_{+}^{D}(Y), F_{-}^{D}(X)\right\rangle .
\end{aligned}
$$

Consequently the following are equivalent:

(i) $F^{D}$ is either selfdual or antiselfdual.

(ii) $B=0$ and either $\mathrm{scal}^{D}=0$ or $D$ is exact.

Proof. On an Einstein-Weyl 4-manifold, $r^{D}=\frac{1}{24} \mathrm{scal}^{D}-\frac{1}{2} F^{D}$ and so the Cotton-York tensor is:

$$
C_{U, X}^{D} Y=\frac{1}{24}\left(D_{U} \operatorname{scal}^{D}\langle X, Y\rangle-D_{X} \operatorname{scal}^{D}\langle U, Y\rangle\right)+\frac{1}{2} D_{Y} F^{D}(U, X)
$$

where I have used the fact that $d F^{D}=0$. Consequently, using 3.1 and 3.2,

$$
\begin{aligned}
B(X, Y) & =\frac{1}{24}\left(D_{X, Y}^{2} \operatorname{scal}^{D}+D_{Y, X}^{2} \operatorname{scal}^{D}\right) \\
+ & \frac{1}{4}\left(F^{D}\left(R_{X, e_{i}}^{D} e_{i}, Y\right)+F^{D}\left(R_{Y, e_{i}}^{D} e_{i}, X\right)+F^{D}\left(e_{i}, R_{X, e_{i}}^{D} Y+R_{Y, e_{i}}^{D} X\right)\right) .
\end{aligned}
$$

After some work, the curvature terms reduce to just $-\left\langle F^{D}(X), F^{D}(Y)\right\rangle+$ $\frac{1}{2}\left|F^{D}\right|^{2}\langle X, Y\rangle$. Writing $F^{D}=F_{+}^{D}+F_{-}^{D}$ gives the formula in the theorem, since the symmetric tracefree part of $\left\langle F_{+}^{D}(X), F_{+}^{D}(Y)\right\rangle$ is zero, and similarly for $F_{-}^{D}$. The equivalence of (i) and (ii) follows from this formula, together with the fact that Einstein-Weyl manifolds with (anti)selfdual Faraday satisfy $D$ scal $^{D}=0$ (see [14] or 3.3). To see that (ii) implies (i), observe that $D \mathrm{scal}^{D}=0$ and $B=0$ together imply that one of $F_{+}^{D}, F_{-}^{D}$ must vanish at each point, since $\Lambda_{+}^{2} T^{*} M \otimes \Lambda_{-}^{2} T^{*} M \cong L^{-2} S_{0}^{2} T^{*} M$. Now, Einstein-Weyl manifolds are real analytic [24], [25] and so (assuming $M$ is connected), $F^{D}$ must be everywhere selfdual or antiselfdual (or both, i.e., zero).

In the compact case, we only need one of the conditions $B=0$ or scal ${ }^{D}=$ 0 to ensure that $F^{D}=0$. It is natural to ask whether something similar holds in general. However, Bonneau [4] has recently given examples of U(2)symmetric Einstein-Weyl structures with vanishing scalar curvature, for which 
the Faraday 2-form is not (anti)selfdual. On the other hand, Bach flat EinsteinWeyl structures have not been studied locally. Are they necessarily scalar flat or Einstein? Equivalently, must $F^{D}$ be (anti)selfdual?

\section{The Weyl tensor and the Faraday 2-form}

Theorem 4.4 implies in particular that on a scalar flat Einstein-Weyl manifold with selfdual Weyl tensor, the Faraday 2-form is either selfdual or antiselfdual, since such manifolds are Bach flat. Now selfduality of the Weyl tensor is a much stronger assumption than Bach flatness, and so one might hope to improve this result in two ways: firstly, by removing the assumption of scalar flatness; and secondly, by showing that the Faraday 2 -form is in fact selfdual. In this section I will prove such a result:

THeorem 5.1. Let $M$ be an Einstein-Weyl 4-manifold with selfdual Weyl tensor. Then the Faraday 2-form is also selfdual.

This is similar to the following theorem of Eastwood and Tod:

Theorem 5.2. [9] Let $M$ be an Einstein-Weyl $n$-manifold $(n \geq 4)$ with vanishing Weyl tensor. Then the Faraday 2-form also vanishes.

I want to explain how Eastwood and Tod's result fits into the framework of twisting complexes of differential operators, and show that in four dimensions, one can split the argument into its selfdual and antiselfdual parts. Therefore, as well as proving 5.1, I will provide another proof of 5.2.

The reader may have noticed that the underlying ideas behind many of the results in this paper are representation theoretic in nature; that is, they involve the representation theory of the orthogonal group and the theory of conformally invariant differential operators. I would now like to make these ideas more explicit, since the proofs of 5.1 and 5.2 are quite straightforward from this point of view, whereas the detailed calculations are not so simple.

Irreducible representations of $\mathrm{SO}(n)$ may be described by $l$-tuples of integers, where $n=2 l$ or $2 l+1$, and I will use these $l$-tuples to describe the associated vector bundles on a Riemannian manifold $M$ [10], [11]. For example $(1,0, \ldots)$ is the (co)tangent bundle, $(1,1, \ldots)$ is the bundle of 2 -forms and $(2,0, \ldots)$ is the bundle of symmetric traceless 2 -tensors. Here the dots indicate that the remaining integers are all zero. The integers are nonincreasing, and are usually all positive, in which case the corresponding tensors have rank equal to the sum of the integers. In four dimensions (and any even dimension), the last integer may be negative: for instance, the selfdual and antiselfdual 2 -forms are denoted by $(1,+1)$ and $(1,-1)$. 
If $M$ is conformal rather than Riemannian, the weight must also be specified. I will do this by prefixing the $l$-tuple by the weight, separated by a vertical bar $\mid$. Thus $(-2 \mid 1,+1)$ denotes the bundle of selfdual 2-forms with their usual weight of -2 . This notation appears ad hoc, but in fact these $(l+1)$-tuples describe the irreducible representations of the parabolic subgroup of $\mathrm{SO}(n+1,1)$ associated with conformal geometry [1]: this consists of those elements of $\mathrm{SO}(n+1,1)$ which stabilise a chosen null line in the null cone $S^{n}$ of $R^{n+1,1}$ (the model geometry on which all conformal manifolds are based).

On a conformally flat manifold there is a complex

$$
(1 \mid 1,1, \ldots) \stackrel{\text { Twist }}{\longrightarrow}(0 \mid 2,1, \ldots) \stackrel{\text { CLanc }}{\longrightarrow}(-1 \mid 2,2, \ldots)
$$

of conformally invariant first order differential operators. The three bundles here are the 2-forms of weight 1 , the weightless Cotton-York tensors and the Weyl tensors of weight -1 . The first operator is a kind of twistor operator, and the second is the (conformal) Lanczos potential operator. This complex is the beginning of the Bernstein-Gelfand-Gelfand resolution of the kernel of Twist [1], which (locally, or on $S^{n}$ ) is the representation $(1,1,1,0, \ldots)$ of $\mathrm{SO}(n+1,1)$.

Twisting this complex by a Weyl derivative gives:

$$
(-2 \mid 1,1, \ldots) \stackrel{\text { Twist }^{D}}{\longrightarrow}(-3 \mid 2,1, \ldots) \stackrel{\mathrm{CLanc}^{D}}{\longrightarrow}(-4 \mid 2,2, \ldots) .
$$

This will no longer be a complex in general: $\mathrm{CLanc}^{D} \circ \mathrm{Twist}^{D}$ will be given (up to a nonzero constant) by tensoring a 2-form with $F^{D}$ and projecting onto $(-4 \mid 2,2, \ldots)$. Now suppose that $D$ is Einstein-Weyl, and apply these operators to $F^{D}$ itself.

Since $D$ is Einstein-Weyl, Twist ${ }^{D} F^{D}$ is the Cotton-York tensor of $D$, which vanishes, since $W=0$. Therefore $0=\operatorname{CLanc}^{D}\left(\operatorname{Twist}^{D} F^{D}\right)=\operatorname{pr}\left(F^{D} \otimes F^{D}\right)$, where pr denotes projection onto $(-4 \mid 2,2, \ldots)$ (up to a nonzero constant). It easily follows that $F^{D}$ is zero.

The same argument applied to half of the sequence proves the four dimensional result. If $W^{-}$vanishes, then the sequence

$$
(1 \mid 1,-1) \stackrel{\text { Twist }}{\longrightarrow}(0 \mid 2,-1) \stackrel{\text { CLanc }}{\longrightarrow}(-1 \mid 2,-2)
$$

is a complex [3], [17]. Twisting this with $D$ and applying it to $F_{-}^{D}$ shows that $F_{-}^{D}=0$.

The reader may prefer to regard the above as a sketch, rather than a full proof. The detailed proof is more complicated, because the operators and bundles involved are complicated. In four dimensions, it is perhaps simpler to use spinor methods, since the irreducible representations of $\mathrm{SO}(4)$ are very easily 
described in terms of spinors. Nevertheless, in order to see the four dimensional result in the higher dimensional context, I shall proceed with brutal tensor methods.

First of all, here are the operators $\operatorname{Twist}^{D}$ and $\mathrm{CLanc}^{D}$ :

$$
\begin{aligned}
\left(\operatorname{Twist}^{D} \phi\right)_{X, Y} U= & D_{U} \phi(X, Y)-\frac{1}{3}\left(d^{D} \phi\right)(U, X, Y) \\
& -\frac{1}{n-1}\left(\langle U, X\rangle \delta^{D} \phi(Y)-\langle U, Y\rangle \delta^{D} \phi(X)\right) \\
\left\langle\left(\operatorname{CLanc}^{D} \mathscr{C}_{X, Y} U, V\right\rangle=\right. & \frac{1}{2}\left(D_{U} \mathscr{C}_{X, Y} V-D_{V} \mathscr{C}_{X, Y} U+D_{X} \mathscr{C}_{U, V} Y-D_{Y} \mathscr{C}_{U, V} X\right) \\
& -\frac{1}{n-2}\left(\langle U, X\rangle \operatorname{Sdiv}^{D} \mathscr{C}(V, Y)\right. \\
& -\langle U, Y\rangle \operatorname{Sdiv}^{D} \mathscr{C}(V, X)-\langle V, X\rangle \operatorname{Sdiv}^{D} \mathscr{C}(U, Y) \\
& \left.+\langle V, Y\rangle \operatorname{Sdiv}^{D} \mathscr{C}(U, X)\right),
\end{aligned}
$$

where $\operatorname{Sdiv}^{D} \mathscr{C}(U, X)=\frac{1}{2}\left(D_{e_{i}} \mathscr{C}_{e_{i}, U} X+D_{e_{i}} \mathscr{C}_{e_{i}, X} U\right)$. In these formulae, $\phi$ and $\mathscr{C}$ can have any weight $w$, but $\operatorname{Twist}^{D}$ is only conformally invariant (independent of $D$ ) when $w=1$, whereas $\operatorname{CLanc}^{D}$ is only conformally invariant when $w=0$.

To check the conformal invariance, and to find out what happens when $\phi$ and $\mathscr{C}$ have the "wrong" weight, suppose that $\tilde{D}=D+\gamma$ on $L^{1}$. Then, if $\phi$ has weight $w, \tilde{D}_{U} \phi(X, Y)=D_{U} \phi(X, Y)+w \gamma(U) \phi(X, Y)-\phi((\gamma \triangle U) X, Y)-$ $\phi(X,(\gamma \triangle U) Y)$.

If we now use this in the formula for Twist ${ }^{\tilde{D}}$ we obtain

$$
\begin{aligned}
&\left(\operatorname{Twist}^{\tilde{D}} \phi\right)_{X, Y} U-\left(\operatorname{Twist}^{D} \phi\right)_{X, Y} U \\
&=(w-1)\left(\frac{2}{3} \gamma(U) \phi(X, Y)-\frac{1}{3}(\gamma(X) \phi(Y, U)-\gamma(Y) \phi(X, U))\right. \\
&\left.\quad-\frac{1}{n-1}(\langle U, X\rangle \phi(\gamma, Y)-\langle U, Y\rangle \phi(\gamma, X))\right) .
\end{aligned}
$$

A similar formula holds for $\mathrm{CLanc}^{D}$. Such formulae verify special cases of the general theory of conformally invariant first order differential operators due to Fegan [10]. Equation (2) is another instance of this.

Since the principal symbols of $\mathrm{CLanc}^{D}$ and $\mathrm{Twist}^{D}$ are canonically defined in terms of the conformal structure, the composite $\operatorname{CLanc}^{D} \circ$ Twist $^{D} \phi$ is obtained by taking the second derivative $D^{2} \phi$ and applying an equivariant bundle homomorphism $T^{*} M \otimes T^{*} M \otimes(w \mid 1,1, \ldots) \rightarrow(w-2 \mid 2,2, \ldots)$. Now the representation $(w-2 \mid 2,2, \ldots)$ does not appear in the decomposition of the tensor product of $(w \mid 1,1, \ldots)$ with $S^{2} T^{*} M=(-2 \mid 0, \ldots)+(-2 \mid 2,0, \ldots)$, 
and so $\mathrm{CLanc}^{D} \circ \mathrm{Twist}^{D}$ is a zero order, given by the action of the curvature of $D$. More precisely, $\mathrm{CLanc}^{D} \circ \mathrm{Twist}^{D} \phi$ is obtained by applying the composite $\Lambda^{2} T^{*} M \otimes \mathfrak{C D}(T M) \otimes(w \mid 1,1, \ldots) \rightarrow \Lambda^{2} T^{*} M \otimes(w \mid 1,1, \ldots) \rightarrow(w-2 \mid 2,2, \ldots)$

to $R^{D} \otimes \phi$ where the first map is the natural action of $\operatorname{co}(T M)$, and the second is projection. Again since $(w-2 \mid 2,2, \ldots)$ is not a subrepresentation of $S^{2} T^{*} M \otimes(w \mid 1,1, \ldots)$, the symmetric part of the Ricci tensor of $D$ does not contribute. Therefore, on a conformally flat manifold CLanc ${ }^{D^{0}}$ 。 Twist ${ }^{D^{0}}=0$ for any closed Weyl derivative $D^{0}$, and in particular, the conformally invariant composite CLanc $\circ$ Twist is zero on $(1 \mid 1,1, \ldots)$. In four dimensions Twist ${ }^{D}$ and $\mathrm{CLanc}^{D}$ split into separate operators on the selfdual and antiselfdual parts of the corresponding tensors. The composite CLanc ${ }^{D^{0}} \circ \mathrm{Twist}^{D^{0}}$ will be zero on $(w \mid 1,-1)$ provided $W^{-}=0$. The representation theoretic reasons behind this are well known: on antiselfdual 2-forms, Twist is the operator $\bar{D}_{2}$ in [3], section $13 \mathrm{C}$.

Therefore we only need to compute the contribution of Faraday curvature to the composite $\operatorname{CLanc}^{D} \circ \operatorname{Twist}^{D}$ on $(w \mid 1,1, \ldots)$. This can be done by writing $D=D^{0}+\omega$ with $D^{0}$ exact, then applying CLanc $D^{0}$ to Twist ${ }^{D} \phi$ and looking for the $D^{0} \omega$ terms. The $D^{0} \omega$ terms in $\left(D_{V}^{0} \text { Twist }^{D} \phi\right)_{X, Y} U$ are

$$
\begin{aligned}
(w-1)\left(\frac{2}{3} D_{V}^{0} \omega(U) \phi(X, Y)-\frac{1}{3}\left(D_{V}^{0} \omega(X) \phi(Y, U)-D_{V}^{0} \omega(Y) \phi(X, U)\right)\right. \\
\left.-\frac{1}{n-1}\left(\langle U, X\rangle \phi\left(D_{V}^{0} \omega, Y\right)-\langle U, Y\rangle \phi\left(D_{V}^{0} \omega, X\right)\right)\right) .
\end{aligned}
$$

On applying the symmetries defining CLanc ${ }^{0}$, the result has to be a constant multiple of the projection of $d \omega \otimes \phi$ onto $(w-2 \mid 2,2, \ldots)$. Indeed the only reason we need to do the calculation is to check that for $w \neq 1$, the constant multiple is nonzero. This turns out to be the case: the constant is $w-1$.

It remains to apply CLanc ${ }^{D} \circ$ Twist $^{D}$ to $F^{D}$ itself. The result is the projection of $-3 F^{D} \otimes F^{D}$ onto $(-4 \mid 2,2, \ldots)$. As shown by Eastwood and Tod [9], this vanishes iff $F^{D}=0$. In four dimensions, only $F_{-}^{D} \otimes F_{-}^{D}$ contributes to the projection onto $(-4 \mid 2,-2)$. It is immediate that this projection vanishes iff $F_{-}^{D}$ itself vanishes, because $(-4 \mid 2,-2)=S_{0}^{2}(-2 \mid 1,-1)$.

Hence the argument outlined earlier goes through: the formula for Twist ${ }^{D}$, together with the Einstein-Weyl condition and Proposition 3.1, shows that Twist ${ }^{D} F^{D}$ is the Cotton-York tensor of $D$. By the contracted Bianchi identity (3), this vanishes if $W$ vanishes, and similarly, in four dimensions, it is selfdual if $W$ is selfdual. Consequently, $\operatorname{CLanc}^{D}\left(\operatorname{Twist}^{D} F^{D}\right.$ ) vanishes (or is selfdual), which in turn implies that $F^{D}$ vanishes (or is selfdual). The proofs of Theorems 5.1 and 5.2 are therefore complete. 
In [23], Pedersen and Swann studied the twistor theory of Einstein-Weyl structures on a selfdual conformal 4-manifold. They found that they could only give a twistor interpretation of the Einstein-Weyl condition when the Faraday 2 -form was selfdual. Theorem 5.1 shows that their twistor construction in fact covers all Einstein-Weyl structures on a selfdual conformal 4-manifold.

Finally, combining Proposition 5.4 of [23] with Theorem 5.1, we obtain:

Theorem 5.3. Let $D$ be a Weyl derivative on a selfdual conformal 4manifold. Then the following are equivalent:

(i) D is Einstein-Weyl

(ii) Either $D$ is Einstein, or D is the Obata connection of an antiselfdual locally hypercomplex structure on $M$.

The Faraday 2-form $F^{D}$ is then selfdual, and either $F^{D}=0$ or scal ${ }^{D}=0$.

The hypercomplex Einstein-Weyl manifolds are also related to local heterotic geometries [27]. These are Riemannian geometries with torsion, which arise as target spaces for $(4,0)$-supersymmetric nonlinear $\sigma$-models.

\section{The scalar curvature of a compact Einstein-Weyl manifold}

There has been some discussion in the Einstein-Weyl literature on the sign of the scalar curvature. Einstein manifolds have constant scalar curvature, and the sign (positive, zero or negative) has implications for the global topology. On a Weyl manifold, the scalar curvature is a section of $L^{-2}$, and so the only invariant notion of "constant scalar curvature" is the condition $D \mathrm{scal}^{D}=0$, which implies $D$ is exact or scal ${ }^{D}=0$. Nevertheless, it is meaningful to speak of the sign of scal ${ }^{D}$ at each point, since $L^{-2}$ is oriented. Consequently it is natural to ask whether the scalar curvature has constant sign on a compact Einstein-Weyl $n$-manifold. My aim in this section is to prove that this is true when $n \geq 4$ and point out that there are counterexamples when $n=2,3$. This is contrary to some assertions in the literature: the scalar curvature was generally believed to have constant sign in dimensions three and four, but not in higher dimensions [24], [25].

One motivation for including this material here is to clarify (I hope) the situation. Another reason is that the Faraday 2 -form enters in a fundamental way through 3.2:

$$
\Delta^{D} \operatorname{scal}^{D}=-n(n-4)\left|F^{D}\right|^{2} .
$$

The main result of this section will follow by applying the strong (Hopf) maximum principle to this Laplacian. In order to do this, we need to turn scal ${ }^{D}$ into a (weightless) function, by choosing a length scale $\mu$. If $D=D^{\mu}+\omega^{\mu}$ then on $L^{-2}$,

$$
\Delta^{D}=\Delta^{\mu}+(n-6) D_{b \omega^{\mu}}^{\mu}-2 \operatorname{tr} D^{\mu} \omega^{\mu}-2(n-4)\left\langle\omega^{\mu}, \omega^{\mu}\right\rangle .
$$


The gauge $\mu$ is said to be a Gauduchon gauge iff $\operatorname{tr} D^{\mu} \omega^{\mu}=0$. Such a gauge exists uniquely up to homothety if $M$ is compact ([13]— see also [5], [26]), and in any such gauge,

$\Delta^{\mu} \operatorname{scal}^{D}+(n-6) D_{b \omega^{\mu}}^{\mu} \operatorname{scal}^{D}-2(n-4)\left\langle\omega^{\mu}, \omega^{\mu}\right\rangle \operatorname{scal}^{D}=-n(n-4)\left|F^{D}\right|^{2}$.

We can now multiply by $\mu^{2}$ to make scal ${ }^{D}$ into a function.

It immediately follows that if $n>4, \mu^{2} \mathrm{scal}^{D}$ cannot have a negative local minimum unless $\omega^{\mu}=0$ : at a local minimum $\Delta^{\mu} \operatorname{scal}^{D} \geq 0$ and $D_{\mathrm{b} \omega^{\mu}}^{\mu} \operatorname{scal}^{D}=$ 0 , from which it follows that $2\left\langle\omega^{\mu}, \omega^{\mu}\right\rangle \operatorname{scal}^{D} \geq n\left|F^{D}\right|^{2} \geq 0$. (This can also be established using a formula for $\Delta^{\mu} \omega^{\mu}$ given in [14].)

The Hopf maximum principle (see [15]) provides the following refinement of this result.

- If $n \geq 4, \mu^{2} \operatorname{scal}^{D}$ cannot have a nonpositive minimum unless it is constant.

- If $n \leq 4, \mu^{2} \mathrm{scal}^{D}$ cannot have a nonpositive maximum unless it is constant.

Therefore when $n=2,3$ we cannot rule out the possibility that $\mu^{2} \mathrm{scal}^{D}$ has negative minima and positive maxima. On the other hand, for $n \geq 4$ we have that in any Gauduchon gauge, either $\mu^{2} \mathrm{scal}^{D}$ is constant, or its local minima are all positive (and in four dimensions the local maxima are also all positive).

In the compact case, the existence of the Gauduchon gauge, together with the existence of maxima and minima, implies that $\operatorname{scal}^{D}$ has constant sign on Einstein-Weyl $n$-manifolds when $n \geq 4$.

This result is contrary to Corollary 4.4 in [24], where the authors claim that some of their examples have scalar curvature with non-constant sign. The error seems to involve inconsistent choices of the branch of an inverse trigonometric function in the evaluation of the scalar curvature at $\gamma^{-1}\left(c_{1} / 2\right)$.

I finish this section by remarking that the above dichotomy between low dimensions and higher dimensions is genuine in the sense that in two and three dimensions there do exist compact Einstein-Weyl manifolds with scalar curvature of nonconstant sign. This is also contrary to some remarks in the literature (e.g. [25] p. 107, [24] p. 99). I will show here that there are EinsteinWeyl structures with scalar curvature of nonconstant sign on $S^{3}$. Examples also exist on $S^{2}$ and $S^{1} \times S^{1}$ : see [6].

In [25], Pedersen and Tod found Einstein-Weyl structures on $S^{3}$ parameterised by $a, b, c \in \mathrm{R}$ with $a \neq 0$. In his classification of possible compact Einstein-Weyl geometries [26], Tod reobtained these solutions in the follow- 
ing form.

$$
\begin{aligned}
& g=P(v)^{-1} d v^{2}+P(v) d y^{2}+v^{2}\left(d t+C v^{-2} d y\right)^{2} \\
& \omega=2 \lambda v^{2}\left(d t+C v^{-2} d y\right), \\
& D=D^{g}+\omega
\end{aligned}
$$

where

$$
P(v)=-\lambda^{2} v^{4}+A v^{2}+B-C^{2} v^{-2}
$$

and $\lambda, A, B, C$ are arbitrary constants, related to the parameters $a, b, c$ in [25] by:

$$
A=-a^{2}+b^{2}+c^{2}, \quad \lambda^{2} B=a^{2} b^{2}+a^{2} c^{2}-b^{2} c^{2}, \quad \lambda^{4} C^{2}=a^{2} b^{2} c^{2} .
$$

Tod's change of coordinates is well defined provided $b \neq c$ and $b \neq-c$. The scalar curvature is $\mathrm{scal}^{D}=-6 A+3|\omega|^{2}=6\left(a^{2}-b^{2}-c^{2}+2 \lambda^{2} v^{2}\right)$. Also, the range of $\lambda^{2} v^{2}$ when $P(v) \geq 0$ is the interval $\left[b^{2}, c^{2}\right]$. Therefore, for $\left|b^{2}-c^{2}\right|>a^{2}$, the scalar curvature has nonconstant sign.

\section{REFERENCES}

1. Baston, R. J. and Eastwood, M. G., The Penrose Transform, Oxford University Press, Oxford, 1989.

2. Branson, T. P., Second order conformal covariants, Proc. Amer. Math. Soc. 126 (1998), 1031-1042.

3. Besse, A. L., Einstein manifolds, Ergeb. Math. Grenzgeb. (3) 10, 1987.

4. Bonneau, G., Einstein-Weyl structures corresponding to diagonal Kähler Bianchi IX metrics, Class. Quantum Grav. 14 (1997), 2123-2135.

5. Calderbank, D. M. J., and Pedersen, H., Einstein-Weyl geometry, Essays on Einstein manifolds, International Press, Cambridge, 1999.

6. Calderbank, D. M. J., Möbius structures and two dimensional Einstein-Weyl geometry, J. Reine Angew. Math. 504 (1998), 37-53.

7. Cartan, E., Sur une classe d'espaces de Weyl, Ann. Sci. École Norm. Sup. (3) 60 (1943), 1-16.

8. Eastwood, M. G., and Rice, J. W., Conformally invariant operators on Minkowski space and their curved analogues, Comm. Math. Phys. 109 (1987), 207-228

9. Eastwood, M. G., and Tod, K. P., Local constraints on Einstein-Weyl geometries, J. reine angew. Math. 491 (1997), 183-198.

10. Fegan, H. D., Conformally invariant first order differential operators, Quart. J. Math. Oxford 27 (1976), 371-378.

11. Fulton, W., and Harris, J., Representation Theory, Graduate Texts in Mathematics 129, 1991.

12. Gauduchon, P., Connexion canonique et structures de Weyl en géométrie conforme, Preprint (1990).

13. Gauduchon, P., La 1-forme de torsion d'une variété hermitienne compacte, Math. Ann. 267 (1984), 495-518.

14. Gauduchon, P., Structures de Weyl-Einstein, espaces de twisteurs et variétés de type $S^{1} \times S^{3}$, J. Reine Angew. Math. 469 (1995), 1-50. 
15. Gilbarg, D., and Trudinger, N. S., Elliptic Partial Differential Equations of Second Order, Springer-Verlag, Berlin Heidelberg (1977).

16. Higa, T., Curvature tensors and curvature conditions in Weyl geometry, Comment. Math. Univ. Sancti Pauli 43 (1994), 139-153.

17. Hitchin, N. J., Linear field equations on self-dual spaces, Proc. Roy. Soc. London A 370 (1980), 173-191.

18. Hitchin, N. J., Complex manifolds and Einstein equations, in Twistor Geometry and Nonlinear Systems (eds H. D. Doebner and T. D. Palev), Lecture Notes in Math. 970 (1982), 79-99.

19. Jones, P. E., and Tod, K. P., Minitwistor spaces and Einstein-Weyl spaces, Class. Quantum Grav. 2 (1985), 565-577.

20. LeBrun, C. R., Counterexamples to the generalized positive action conjecture, Comm. Math. Phys., $118(1988,591-596$.

21. Madsen, A. B., Einstein-Weyl structures in the conformal classes of LeBrun metrics, Class. Quantum Grav. 14 (1997), 2635-2645.

22. Pedersen, H., Einstein metrics, spinning top motions and monopoles, Math. Ann. 274 (1986), 35-39.

23. Pedersen, H., and Swann, A., Riemannian submersions, four-manifolds and Einstein-Weyl geometry, Proc. London Math. Soc. 66 (1993), 381-399.

24. Pedersen, H., and Swann, A., Einstein-Weyl geometry, the Bach tensor and conformal scalar curvature, J. Reine Angew. Math. 441 (1993), 99-113.

25. Pedersen, H., and Tod, K. P., Three-dimensional Einstein-Weyl geometry, Adv. Math. 97 (1993), 74-109.

26. Tod, K. P., Compact 3-dimensional Einstein-Weyl structures, J. London Math. Soc. 45 (1992), 341-351.

27. Tod, K. P., Local heterotic geometry and self-dual Einstein-Weyl spaces, Class. Quantum Grav. 13 (1996), 2609-2616.

28. Weyl, H., Space, Time, Matter (Translation of the fourth edition of Raum, Zeit, Materie the first edition of which was published in 1918 by Springer, Berlin), Dover, New York (1952).

MATHEMATICS INSTITUTE

UNIVERSITY OF WARWICK

COVENTRY CV4 7AL

ENGLAND
DEPARTMENT OF MATHEMATICS AND STATISTICS

UNIVERSITY OF EDINBURGH

KING'S BUILDINGS, MAYFIELD ROAD

EDINBURGH EH9 3JZ

SCOTLAND

E-mail: davidmjc@maths.ed.ac.uk 